- Original Article

\title{
The Association Between Shift Work and Health Behavior: Findings from the Korean National Health and Nutrition Examination Survey
}

\author{
Myung-Ji Bae, Yun-Mi Song*, Jin-Young Shin, Bo-Young Choi, Jung-Hyun Keum, Eun-Ae Lee \\ Department of Family Medicine, Samsung Medical Center, Sungkyunkwan University School of Medicine, Seoul, Korea
}

\begin{abstract}
Background: Shift workers are increasing worldwide, and various negative health effects of shift work have been reported. This study aimed to evaluate the relationship between shift work and health behavior.

Methods: This cross-sectional study included a total of 11,680 Korean adults (6,061 men and 5,619 women) aged $\geq 20$ years old who participated in the Fifth Korean National Health and Nutrition Examination Survey, 2010-2012. Multiple logistic regression analysis was performed to evaluate the association between shift work and health behavior after adjusting for covariates.

Results: In men, shift work was associated with an increased risk of inadequate sleep (odds ratio [OR], 1.18; 95\% confidence interval [CI], 1.00 to 1.40) compared to day work. In women, shift work was associated with an increased risk of smoking (OR, 1.73; 95\% CI, 1.34 to 2.22) and inadequate sleep (OR, 1.24; 95\% CI, 1.05 to 1.47) compared to day work. In an age-stratified subgroup analysis, female shift workers aged $\geq 50$ years old demonstrated an increased risk of smoking (OR, 5.55; 95\% CI, 3.60 to 8.55), alcohol consumption (OR, 2.22; 95\% CI, 1.53 to 3.23), and inadequate sleep (OR, 1.50; 95\% CI, 1.10 to 2.05 ) compared to female day workers.

Conclusion: Shift work is associated with worse health behavior, and this is most evident in women aged $\geq 50$ years. Targeted strategies to reduce the negative health effects of shift work should be implemented, with consideration of shift workers' demographic characteristics.
\end{abstract}

Keywords: Alcohol Abuse; Health Behavior; Sleep Disorders; Smoking; Work Schedule Tolerance 


\section{INTRODUCTION}

Over the last few decades, globally, the number of shift workers has increased rapidly to keep pace with the increasingly complex societal need for services and operations to be provided around the clock ceaselessly. ${ }^{1)}$ The Korean working condition survey reported that the proportion of employees involved in shift work increased from $7.2 \%$ in 2006 to $10.9 \%$ in 2010. In this survey, the most commonly reported shift work type was the regular two-shift (38.6\%), followed by regular three-shifts (23.9\%), 24-hour rotating shift (14.0\%), irregular two-shift (5.7\%), fixed shift (5.5\%), weekday split-shift (3.6\%), irregular threeshift (2.9\%), and others (5.8\%). $)^{2,3)}$

Owing to the global increase in shift work, its effect on health has garnered increased attention from researchers, various health effects of shift work have been reported: sleep disorders; work-related injuries; digestive diseases such as gastritis and peptic ulcers; cerebrocardiovascular diseases such as ischemic stroke and ischemic heart disease; cancers such as breast cancer, prostate cancer, and colorectal cancer; reproductive outcomes such as preterm birth and natural miscarriage; behavioral disorders in children of shift workers; depressed mood; increased mortality; and low bone mineral density. ${ }^{2)}$ Furthermore, in 2010, the International Agency for Research on Cancer classified night shift work as a probable human carcinogen. ${ }^{4,5}$

Previous studies have suggested several mechanisms for the negative health effects associated with shift work. ${ }^{1,6,7)}$ The circadian rhythm disruption in shift workers may cause a decrease in total sleep time and sleep efficiency, as well as an increase in insomnia prevalence and daytime sleepiness. Such disruptions influence the suprachiasmatic nucleus and endocrine functions, affecting the secretion of hormones such as growth hormone, thyroid hormone, corticosteroids, melatonin, serotonin, gastrin, and pepsinogen. These hormonal imbalances may then result in various clinical manifestations including gastric and duodenal ulcers. ${ }^{1,6,7)}$ Another probable mechanism pertains to the shift workers' health behaviors. Owing to irregular working hours, shift workers are more likely to experience disturbances in their daily routine, and thus have difficulty in maintaining daily health behaviors, increasing their vulnerability to diseases.

Most observational and experimental studies evaluating the association between shift work and health behavior have been conducted in the United States or in the European countries ${ }^{6,8-16)}$ with few reports from Asian countries. ${ }^{17,18)}$ Up to now, several Korean studies have evaluated the relationship between smoking and working conditions or employment status such as permanent or temporary placements, overall work intensity, and weekly working hours. ${ }^{19,20)}$ however, these Korean studies not include shift workers.

Therefore, in the present study, we evaluated the association between shift work and health behaviors such as smoking, alcohol consumption, exercise, and the adequacy of sleep time in the Korean population using data from the fifth the Korean National Health and Nutrition Examination Survey.

\section{METHODS}

\section{Study Subjects}

This study utilized data from the fifth Korean National Health and $\mathrm{Nu}$ trition Examination Survey (KNHANES V). The survey was conducted on a nationwide basis, in a representative sample of South Korean adults and children, using a complex, multi-stage probability sample design, from 2010 to 2012 . The survey consisted of three parts: the health interview survey, the health examination survey, and the nutrition survey health examination to examine the participants' health conditions, socio-demographic characteristics, health behaviors, and nutritional status. ${ }^{21-23)}$ Of the 25,534 participants of the KNHANES V, participants aged $<20$ years $(6,140)$ and participants with missing information on working status, i.e., day work/shift work $(13,465)$ were excluded. A total of 6,061 men and 5,619 women were included in this study for the final analysis.

\section{Study Variables}

There is no internationally standardized definition of shift work, and hence, previous studies show heterogeneity in the definitions used for analysis. We, therefore, classified the type of work based on the participant's answer to a relevant survey question: "Do you usually work in the daytime (6:00 am to 6:00 pm) or other times?" Participants who answered "I usually work in the daytime" were classified as day workers, while participants who reported as being involved in evening shift, night shifts, regular day-night shifts, 24-hour rotating shifts, split shifts, or irregular shifts were classified as shift workers.

Participants who had smoked $\geq 5$ packs of cigarettes (100 units) during their lifetime and were currently smoking either every day or sometimes were classified as 'current smokers,' and the remaining were classified as 'current nonsmokers.'

Alcohol consumption was assessed and classified based on drinking frequency and amount consumed at one time. Male participants who drank $\geq 7$ cups and female participants who drank $\geq 5$ cups more than once a month were categorized as 'high-risk drinkers, and the remaining alcohol drinkers were categorized as 'moderate drinkers.'

Vigorous exercise was defined as physical activity such as jogging, climbing, high-speed cycling or swimming, soccer, basketball, jump rope, squash, tennis (singles), or manual labor involving heavy loads, performed for a duration of $>20$ minutes and at a frequency of $\geq 3$ times per week. Moderate exercise was defined as activities such as lowspeed swimming, tennis (doubles), volleyball, badminton, table tennis, or manual labor involving light loads, for $>30$ minutes and $\geq 5$ times per week. A low-intensity exercise was defined as walking or commuting for $>30$ minutes, $\geq 5$ times per week. Participants who performed vigorous, moderate, or low-intensity exercises were included in the 'physical activity' group, while the remaining were included in the 'physical inactivity' group.

Previous studies have reported that a sleep duration of 7 to 8 hours per day is associated with lower risk of obesity, hypertension, diabetes, myocardial infarction, cerebral vascular accidents, and reduced risk of 
injuries and errors. ${ }^{7)}$ With this reference value, we categorized participants into two sleep groups: adequate sleep group ( 7 to 8 hours per day), or inadequate sleep group ( $\leq 6$ hours or $\geq 9$ hours), respectively.

Based on the highest level of education attained, participants were categorized as 'middle school or lower,' 'high school,' and 'college or higher.'

The monthly average family equivalent income was calculated as total household income divided by the square root of the number of household members. Participants' income level was classified based on monthly average family equivalent income quartiles: 1st (lowest), 2nd, 3rd, and 4th (highest).

Married participants who were currently living with spouse were classified into the 'with spouse' group while never married, divorced, or widowed participants were classified into the 'without spouse' group.

\section{Statistical Analysis}

All estimates were calculated based on the sampling weight provided in the KNHANES V. Continuous variables were compared between the day workers and shift workers using the Student t-test, while the categorical variables were compared using a chi-square test. The association between shift work and health behavior was evaluated using a logistic regression analysis. For multivariable adjusted analysis, age, av- erage working hours per week, education level, income level, and marital status were included in the model as covariates. Analyses were performed for all participants, and also separately for men and women.

To assess the effect of age on the association between shift work and health behavior, we repeated the multiple logistic regression analyses based on three age strata (20-39, 40-49, and 50 years), with adjustment for average working hours per week, education level, income level, and marital status. The effect of age on the association between work type and health behavior was assessed by including an interaction term (work type*age group) in the multiple logistic regression models.

All analyses were conducted using IBM SPSS software for Windows ver. 21.0 (IBM Corp., Armonk, NY, USA). P-values $<0.05$ were considered statistically significant. The study protocol was approved by the Samsung Medical Center institutional review board (SMC 2015-12112).

\section{RESULTS}

\section{General Characteristics of the Study Participants}

In total, 5,041 male day workers, 1,020 male shift workers, 4,709 female day workers, and 910 female shift workers were included in the study. Table 1 shows the weighted distribution of the study participants' gen-

Table 1. Characteristics of study participants according to the type of work

\begin{tabular}{|c|c|c|c|c|c|c|c|}
\hline \multirow[b]{2}{*}{ Characteristic } & \multirow[b]{2}{*}{ Category } & \multicolumn{3}{|c|}{ Male } & \multicolumn{3}{|c|}{ Female } \\
\hline & & $\begin{array}{c}\text { Day work } \\
(\mathrm{N}=5,041)\end{array}$ & $\begin{array}{l}\text { Shift work } \\
(N=1,020)\end{array}$ & P-value & $\begin{array}{c}\text { Day work } \\
(\mathrm{N}=4,709)\end{array}$ & $\begin{array}{l}\text { Shift work } \\
(N=910)\end{array}$ & P-value* \\
\hline Total & & 82.1 & 17.9 & & 82.0 & 18.0 & \\
\hline Age (y) & & $43.8 \pm 0.3$ & $40.4 \pm 0.5$ & $<0.001$ & $44.4 \pm 0.3$ & $39.9 \pm 0.5$ & $<0.001$ \\
\hline \multirow[t]{2}{*}{ Smoking } & Non-smoker or ex-smoker & 56.5 & 53.7 & 0.284 & 95.1 & 90.0 & $<0.001$ \\
\hline & Current smoker & 43.5 & 46.3 & & 4.9 & 10.0 & \\
\hline \multirow[t]{3}{*}{ Alcohol consumption } & Non-drinker & 4.2 & 2.7 & 0.061 & 16.1 & 9.6 & $<0.001$ \\
\hline & Moderate drinker & 32.0 & 35.5 & & 56.9 & 58.0 & \\
\hline & High-risk drinker & 63.8 & 61.8 & & 27.0 & 32.4 & \\
\hline \multirow[t]{2}{*}{ Physical inactivity } & Yes & 50.8 & 59.1 & 0.383 & 54.9 & 52.9 & 0.127 \\
\hline & No & 49.2 & 50.9 & & 45.1 & 47.1 & \\
\hline \multirow[t]{3}{*}{ Average sleep time per day (h) } & $\leq 6$ & 40.9 & 43.4 & 0.010 & 39.2 & 39.3 & 0.009 \\
\hline & $7-8$ & 53.7 & 48.7 & & 53.4 & 49.8 & \\
\hline & $\geq 9$ & 5.4 & 7.9 & & 7.4 & 10.9 & \\
\hline \multirow[t]{4}{*}{ Average work time per week (h) } & $\leq 39$ & 21.3 & 23.6 & $<0.001$ & 39.1 & 50.3 & $<0.001$ \\
\hline & $40-48$ & 36.9 & 21.7 & & 32.9 & 24.0 & \\
\hline & $49-60$ & 27.1 & 25.9 & & 18.0 & 12.0 & \\
\hline & $\geq 61$ & 14.7 & 28.8 & & 10.0 & 13.7 & \\
\hline \multirow[t]{3}{*}{ Education level } & $\leq$ Middle school & 26.9 & 21.3 & $<0.001$ & 40.5 & 24.6 & $<0.001$ \\
\hline & High school & 33.4 & 45.8 & & 30.6 & 39.2 & \\
\hline & $\geq$ College & 39.7 & 32.9 & & 28.9 & 36.2 & \\
\hline \multirow[t]{2}{*}{ Current marital status } & With spouse & 16.3 & 26.4 & $<0.001$ & 28.5 & 40.6 & $<0.001$ \\
\hline & Without spouse & 83.7 & 73.6 & & 71.5 & 59.4 & \\
\hline \multirow[t]{4}{*}{ Quartiles of income } & 1st (lowest) & 13.2 & 8.3 & 0.390 & 17.8 & 10.2 & 0.005 \\
\hline & 2nd & 25.6 & 28.3 & & 25.1 & 27.3 & \\
\hline & $3 r d$ & 29.8 & 33.7 & & 27.8 & 32.0 & \\
\hline & 4th (highest) & 31.4 & 29.7 & & 29.3 & 30.5 & \\
\hline
\end{tabular}

Values are presented as mean \pm standard error or $\%$.

${ }^{*}$ Comparison using a Student t-test for continuous variables and a chi-square test for categorical variables, respectively. 
eral characteristics stratified by sex. On an average, in both sexes, the day workers were older compared to the shift workers $(\mathrm{P}<0.001)$. The distribution of average sleep hours per day was significantly different in men $(\mathrm{P}=0.010)$ and women $(\mathrm{P}=0.009)$, and both the longest $(\geq 9$ hours) and the shortest ( $\leq 6$ hours) sleep time were more prevalent among shift workers than among day workers. The distribution of average work time per week differed between the shift workers and day workers of both sexes $(\mathrm{P}<0.001)$, and both the longest $(>60$ hours $)$ and shortest ( $<40$ hours) work time per week were more prevalent among shift workers than among day workers. The highest education level also differed between shift workers and day workers in both sexes. In men, the proportion of participants with more than college level education was higher in day workers, whereas the opposite was observed for women. Shift workers of both sexes were more likely to be unmarried than day workers $(\mathrm{P}<0.001)$. Male shift workers did not differ from their day-working counterparts in their income level, whereas female shift workers had significantly different income level compared to their day-working counterparts. Furthermore, the distribution of current smoking, high-risk drinking, and exercise level behaviors did not differ between male day workers and shift workers. However, female shift workers were more likely to be current smokers $(\mathrm{P}<0.001)$ and high-

Table 2. Association between shift work and health behaviors in Korean men

\begin{tabular}{|c|c|c|c|}
\hline Variable & Age group & $\begin{array}{l}\text { Crude OR } \\
(95 \% \mathrm{Cl})\end{array}$ & $\begin{array}{l}\text { Adjusted OR } \\
(95 \% \mathrm{Cl})^{\star}\end{array}$ \\
\hline \multicolumn{4}{|c|}{ Current smoking } \\
\hline & Overall & $1.11(0.94-1.32)$ & $1.00(0.84-1.19)$ \\
\hline & $20-39$ y & $1.04(0.80-1.36)$ & $1.11(0.84-1.47)$ \\
\hline & $40-49$ y & $1.19(0.86-1.64)$ & $1.24(0.89-1.72)$ \\
\hline & $\geq 50 y$ & $0.97(0.74-1.29)$ & $0.90(0.67-1.22)$ \\
\hline & $\mathrm{P}$ for interaction ${ }^{\dagger}$ & $<0.001$ & $<0.001$ \\
\hline \multicolumn{4}{|c|}{ High-risk drinking ${ }^{\ddagger}$} \\
\hline & Overall & $0.94(0.80-1.11)$ & $0.85(0.71-1.01)$ \\
\hline & $20-39 y$ & $0.91(0.70-1.18)$ & $0.88(0.67-1.15)$ \\
\hline & $40-49$ y & $0.92(0.63-1.34)$ & $0.93(0.63-1.37)$ \\
\hline & $\geq 50 y$ & $0.85(0.65-1.11)$ & $0.85(0.64-1.12)$ \\
\hline & $\mathrm{P}$ for interaction ${ }^{\dagger}$ & $<0.001$ & $<0.001$ \\
\hline \multicolumn{4}{|c|}{ Physical inactivity } \\
\hline & Overall & $1.11(0.94-1.31)$ & $1.00(0.84-1.19)$ \\
\hline & $20-39 y$ & $1.21(0.93-1.58)$ & $0.99(0.74-1.33)$ \\
\hline & $40-49$ y & $1.00(0.71-1.41)$ & $1.00(0.70-1.43)$ \\
\hline & $\geq 50 y$ & $0.94(0.72-1.24)$ & $087(0.66-1.16)$ \\
\hline & $\mathrm{P}$ for interaction ${ }^{\dagger}$ & $<0.001$ & $<0.001$ \\
\hline \multicolumn{4}{|c|}{ Inadequate sleep } \\
\hline & Overall & $1.22(1.04-1.44)$ & $1.18(1.00-1.40)$ \\
\hline & $20-39 y$ & $1.41(1.09-1.82)$ & $1.39(1.06-1.81)$ \\
\hline & $40-49 y$ & $1.07(0.76-1.52)$ & $1.01(0.71-1.43)$ \\
\hline & $\geq 50 y$ & $1.08(0.84-1.40)$ & $1.07(0.81-1.40)$ \\
\hline & $\mathrm{P}$ for interaction ${ }^{\dagger}$ & 0.017 & $<0.001$ \\
\hline
\end{tabular}

$\mathrm{OR}$, odds ratio; $\mathrm{Cl}$, confidence interval.

*Adjusted for age, average work hours per week, education level, income level, and marriage status in multiple logistic regression analysis. Age was not adjusted for the age-stratified subgroup analysis. ${ }^{\dagger}$ Assessed by including an interaction term (work type*age group) in the multiple logistic regression analysis. "For this analysis, nondrinkers and moderate drinkers were combined into one reference group. risk drinkers $(\mathrm{P}<0.001)$ compared to their day working counterparts; however, the exercise habit did not differ significantly between them.

\section{Association between Shift Work and Health Behavior}

Table 2 shows the association between work type and health behavior in men. There were no statistically significant associations between work type and health behaviors such as smoking, alcohol consumption, and exercise despite adjusting for covariates. Furthermore, an analysis of age-stratified data also did not reveal any significant associations between work type and smoking, alcohol consumption, or exercise habit. However, male shift workers were 1.18 times more likely to have inadequate sleep than male day workers were. A subgroup analysis showed that the 20-39 years old shift workers were 1.39 times (95\% confidence interval [CI], 1.06 to 1.81) more likely to have inadequate sleep compared to the day workers.

Table 3 shows the association between work type and health behaviors in women. Compared with the day workers, shift workers had an increased risk of smoking (odds ratio [OR], 1.73; 95\% CI, 1.34 to 2.22) and inadequate sleep (OR, 1.24; 95\% CI, 1.05 to 1.47), after adjusting for covariates. Subgroup analysis by age showed that the $\geq 50$-years-old shift workers had an increased risk of smoking (OR, 5.55; 95\% CI, 3.60

Table 3. Association between shift work and health behaviors in Korean women

\begin{tabular}{|c|c|c|c|}
\hline Variable & Age group & $\begin{array}{l}\text { Crude OR } \\
(95 \% \text { Cl) }\end{array}$ & $\begin{array}{c}\text { Adjusted OR } \\
(95 \% \mathrm{Cl})^{\star}\end{array}$ \\
\hline \multicolumn{4}{|c|}{ Current smoking } \\
\hline & Overall & $1.89(1.41-2.53)$ & $1.73(1.34-2.22)$ \\
\hline & $20-39$ y & $1.42(0.97-2.10)$ & $1.39(0.94-2.08)$ \\
\hline & $40-49$ y & $1.12(0.51-2.47)$ & $1.19(0.53-2.67)$ \\
\hline & $\geq 50 y$ & $4.20(2.40-7.36)$ & $5.55(3.60-8.55)$ \\
\hline & $P$ for interaction ${ }^{\dagger}$ & $<0.001$ & $<0.001$ \\
\hline \multicolumn{4}{|c|}{ High-risk drinking ${ }^{\ddagger}$} \\
\hline & Overall & $1.32(1.08-1.62)$ & $1.03(0.83-1.27)$ \\
\hline & $20-39 y$ & $1.05(0.79-1.39)$ & $0.93(0.70-1.25)$ \\
\hline & $40-49 y$ & $0.96(0.63-1.46)$ & $0.98(0.64-1.49)$ \\
\hline & $\geq 50 y$ & $2.35(1.57-3.53)$ & $2.22(1.53-3.23)$ \\
\hline & $P$ for interaction ${ }^{\dagger}$ & $<0.001$ & $<0.001$ \\
\hline \multicolumn{4}{|c|}{ Physical inactivity } \\
\hline & Overall & $1.16(0.97-1.38)$ & $1.03(0.86-1.23)$ \\
\hline & $20-39 y$ & $1.48(1.11-1.97)$ & $1.27(0.95-1.70)$ \\
\hline & $40-49$ y & $0.73(0.53-1.02)$ & $0.72(0.52-1.01)$ \\
\hline & $\geq 50 y$ & $1.12(0.83-1.50)$ & $1.08(0.80-1.45)$ \\
\hline & $P$ for interaction ${ }^{\dagger}$ & 0.005 & 0.005 \\
\hline \multicolumn{4}{|c|}{ Inadequate sleep } \\
\hline & Overall & $1.15(0.98-1.36)$ & $1.24(1.05-1.47)$ \\
\hline & $20-39$ y & $1.24(0.97-1.59)$ & $1.22(0.94-1.59)$ \\
\hline & $40-49 y$ & $1.05(0.75-1.47)$ & $1.03(0.73-1.45)$ \\
\hline & $\geq 50 y$ & $1.30(0.96-1.78)$ & $1.50(1.10-2.05)$ \\
\hline & $P$ for interaction ${ }^{\dagger}$ & 0.095 & $<0.001$ \\
\hline
\end{tabular}

OR, odds ratio; $\mathrm{Cl}$, confidence interval.

*Adjusted for age, average work hours per week, education level, income level, and marriage status in multiple logistic regression analysis. Age was not adjusted for the age-stratified subgroup analysis. ${ }^{\dagger}$ Assessed by including an interaction term (work type*age group) in the multiple logistic regression analysis. "For this analysis, nondrinkers and moderated drinkers were combined into one reference group. 
to 8.55 ), alcohol consumption (OR, 2.22; 95\% CI, 1.53 to 3.23), and inadequate sleep (OR, 1.50; 95\% CI, 1.10 to 2.05) after adjusting for covariates. There was no statistically significant association between work type and health behavior in the other age groups.

\section{DISCUSSION}

In this cross-sectional study of the Korean adult population, we found that shift work was associated with negative health behavior such as smoking, alcohol consumption, and inadequate sleep, although the degree of association differed based on the participants' sex and age.

People may be more prone to smoking during shift work to relieve stress and counter sleepiness and tiredness. Indeed, many previous studies have reported a higher prevalence of smoking among shift workers. ${ }^{8-11,13,17,24)}$ A study of male blue-collar workers by Knutsson et al. ${ }^{8)}$ revealed that a higher proportion of shift workers were current smokers (shift workers, $54 \%$ vs. day workers, $39 \%$ ). Fujino et al. ${ }^{17)}$ also reported that the Japanese male shift workers had a higher current smoking rate (relative risk ratio [RR], 2.50; $95 \% \mathrm{CI}, 1.39$ to 4.51 ). Consistent with these findings, shift work has also been associated with a higher amount of tobacco consumption in female nurses. ${ }^{10)}$ A study by Trinkoff and Storr ${ }^{13)}$ reported that a night shift of $>8$ hours was associated with a higher current smoking rate in nurses (OR, 1.62; 95\% CI, 1.14 to 2.31). Similarly, another study by Knutsson and Nilsson ${ }^{9)}$ found that shift workers had an increased risk of current smoking (OR 1.3; 95\% CI, 1.1 to 1.6). In a follow-up study by van Amelsvoort et al., ${ }^{12)}$ during the 1-year follow-up period, the proportion of smokers, as well as the number of cigarettes smoked per day by the smokers, increased more for the shift workers compared to the day workers. In another prospective study, van Amelsvoort et al. ${ }^{11)}$ reported that shift workers were more likely to start smoking than their day-working counterparts.

Interestingly, in our study, only the female shift workers were more likely to be current smokers, and the increased risk of smoking was confined to women aged $\geq 50$ years. A possible explanation for this finding could be the difference in smoking prevalence of the studied populations, although we did not test this assumption in our study. The high prevalence ( $>50 \%$ in 2010-2012) of smoking among Korean men suggests that they are more likely to be exposed to smoking. ${ }^{19)}$ Consequently, the common reasons for shift workers to start smoking such as fatigue, or stress, may be less influential in the Korean men compared with other population with lower smoking prevalence. On the other hand, the shift work-related factors may have a greater influence on smoking habit of women than that of men because smoking prevalence is relatively lower among Korean women. ${ }^{19)}$

Shift workers may have a lower desire to consume alcohol due to lethargy and fatigue, or may be more vulnerable to alcohol consumption due to shift work-related stress. Trinkoff and Storr ${ }^{13)}$ observed that both night shifts with longer working time (>8 hours: OR, 1.40; 95\% CI, 1.00 to 1.98 ) and rotating shifts with longer working time (>8 hours: OR, 1.52; 95\% CI, 1.04 to 2.22) were associated with a higher alcohol use in nurses compared to day shifts with shorter working time $(\leq 8$ hours). Fujino et al. ${ }^{17)}$ reported an increased risk of habitual drinking (RR, 2.94; 95\% CI, 1.62 to 5.32), especially ethanol consumption ( $\geq 46$ g/d: RR, 6.56; 95\% CI, 2.09 to 20.56), in Japanese male shift workers. In our study, however, we observed statistically significant difference in alcohol consumption between day and shift workers only for women aged $\geq 50$ years. Similar to the smoking behavior, the high prevalence of alcohol consumption among the male participants across all age groups ( $64.8 \%$ in $20-39$ years, $57.4 \%$ in $40-49$ years, and $51.4 \%$ in $\geq 50$ years) and younger women (34.7\% in $20-39$ years and $24.4 \%$ in $40-49$ years vs. $12.1 \%$ in $\geq 50$ years) may also be a result of a weaker-than-expected influence of shift work on alcohol consumption.

In a study by Nakamura et al., ${ }^{18)}$ lack of exercise was reported in a higher proportion of shift workers (69\%) compared to day workers (50\%), and only approximately $10 \%$ of the shift workers exercised at least once a week. Bushnell et al. ${ }^{24)}$ also reported that 'a lack of exercise' was associated with rotating shift schedules. Lower prevalence of exercise in shift workers may be due to excessive exertion from work or difficulty in scheduling regular exercise. However, in our study, the exercise prevalence did not differ significantly between the day and shift workers. This lack of significant difference did not change when analysis was repeated after excluding the 'low-intensity' exercise subgroup from the 'exercise group' (data not shown).

A previous study has reported that morning shift was associated with shorter total sleep time and increased fatigue or the feeling of inadequate rest. ${ }^{14)}$ In another study, shift work was associated with greater difficulty in falling asleep as well as a higher risk of sleepiness at work. ${ }^{15)}$ Pilcher et al. ${ }^{25)}$ reported that the type of shift work and the speed of shift rotation influenced the length of sleep, resulting in both increase and decrease in sleep duration. Other studies have associated shift work with sleep disturbances such as insomnia, or excessive sleepiness. ${ }^{6,16)}$ Consistent with previous findings, we also observed a higher proportion of inadequate sleep in the shift workers of both sexes. It is plausible that this irregular sleep time may exert undesirable effects on the shift workers' sleep health. Interestingly, we observed that the association between shift work and inadequate sleep was especially evident in 20-39-year-old men and in $\geq 50$-year-old women. However, we could not compare this finding, as other studies have not reported an age-related effect on this association. In addition, we cannot adequately explain this age-specific association in our study.

In this study, the higher prevalence of smoking in shift workers was confined to women. Moreover, the prevalence of smoking, alcohol consumption, and inadequate sleep in the shift workers was also much higher for the female shift workers aged $\geq 50$ years. Several physical and environmental characteristics of women may explain these results. First, women tend to be weaker in terms of basic physical strength than men and may thus feel more fatigue or stress during shift work. Second, women are more vulnerable to hormone imbalances due to the shift work compared to men, and can therefore experience more exhaustion and emotional fluctuations. Third, as is evident from the Korean statistical information, many working women have more domestic duties than men do. ${ }^{26)}$ Taken together, women may have a 
higher workload that may lead to greater tiredness and exhaustion, as well as emotional stress compared to men. These reasons may explain why the shift work associated unhealthy behaviors are more evident in the women than in the men.

There have been many studies evaluating the relationship between age and shift work. Younger people tend to show better performance compared to older people during extended shift work. Older people are more vulnerable to the changes in circadian rhythm, which results in increased alteration in the quality as well as the quantity of sleep. The older people are more likely to have sleep disorders related to shift work, and may have a decreased ability to recover following shift work compared to younger people. ${ }^{27-30)}$ Despite these findings, to the best of our knowledge, no previous studies have conducted an age-stratified analysis as we report here.

Our study has some limitations. First, our study bears the innate limitations of a cross-sectional study, and we could therefore not confirm the causal effect of shift work on health behavior. Second, we could not include several informative work-related factors such as the type of shift work, labor intensity, type of work, commuting time, and work-related stress, in our analysis due to the lack of data.

In conclusion, this cross-sectional study of a representative sample of Korean adults found that shift work was associated with worse health behavior. Shift work was associated with inadequate sleep in both sexes, and with higher smoking prevalence in women. Age-stratified analysis showed that the association was especially evident in older women. Our findings highlight the importance of implementing active strategies to reduce the negative health behavior in shift workers to prevent/decrease adverse health outcome, and that such strategies should consider the shift workers' demographic characteristics.

\section{CONFLICT OF INTEREST}

No potential conflict of interest relevant to this article was reported.

\section{ACKNOWLEDGMENTS}

This research was supported by the Basic Science Research Program through the National Research Foundation of Korea (NRF) funded by the Ministry of Science, ICT, and future Planning (2014R1A2A2A01002705).

\section{REFERENCES}

1. Rosa RR, Colligan MJ. Plain language about shiftwork. Washington (DC): U.S. Department of Health and Human Services, National Institute for Occupational Safety and Health; 1997.

2. Wang JH, Lee G, Song JT, Kwon J, Choi H, Jung-Choi K, et al. The association between shift work and bone mineral density: analysis of 20082009 Korean National Health and Nutrition Examination Survey. Korean J Occup Environ Med 2012;24:274-86.

3. Occupational Safety and Health Research Institute. 2006 Korean working condition survey report. Ulsan: Occupational Safety and Health Research Institute; 2006.
4. International Agency for Research on Cancer. IARC monographs-shift work. Lyon: International Agency for Research on Cancer; 2010.

5. International Agency for Research on Cancer. Agents classified by IARC monographs. Lyon: International Agency for Research on Cancer; 2010.

6. Drake CL, Roehrs T, Richardson G, Walsh JK, Roth T. Shift work sleep disorder: prevalence and consequences beyond that of symptomatic day workers. Sleep 2004;27:1453-62.

7. Colten HR, Altevogt BM. Sleep disorders and sleep deprivation an unmet public health problem. Washington (DC): National Academies; 2006.

8. Knutsson A, Akerstedt T, Jonsson BG. Prevalence of risk factors for coronary artery disease among day and shift workers. Scand J Work Environ Health 1988;14:317-21.

9. Knutsson A, Nilsson T. Tobacco use and exposure to environmental tobacco smoke in relation to certain work characteristics. Scand J Soc Med 1998;26:183-9.

10. Kivimaki M, Kuisma P, Virtanen M, Elovainio M. Does shift work lead to poorer health habits?: a comparison between women who had always done shift work with those who had never done shift work. Work Stress 2001;15:3-13.

11. Van Amelsvoort LG, Jansen NW, Kant I. Smoking among shift workers: more than a confounding factor. Chronobiol Int 2006;23:1105-13.

12. Van Amelsvoort LG, Schouten EG, Kok FJ. Impact of one year of shift work on cardiovascular disease risk factors. J Occup Environ Med 2004;46:699-706.

13. Trinkoff AM, Storr CL. Work schedule characteristics and substance use in nurses. Am J Ind Med 1998;34:266-71.

14. Akerstedt T, Kecklund G, Selen J. Early morning work: prevalence and relation to sleep/wake problems: a national representative survey. Chronobiol Int 2010;27:975-86.

15. Akerstedt T, Nordin M, Alfredsson L, Westerholm P, Kecklund G. Sleep and sleepiness: impact of entering or leaving shiftwork: a prospective study. Chronobiol Int 2010;27:987-96.

16. Ingre $M$, Akerstedt T. Effect of accumulated night work during the working lifetime, on subjective health and sleep in monozygotic twins. J Sleep Res 2004;13:45-8.

17. Fujino Y, Iso H, Tamakoshi A, Inaba Y, Koizumi A, Kubo T, et al. A prospective cohort study of shift work and risk of ischemic heart disease in Japanese male workers. Am J Epidemiol 2006;164:128-35.

18. Nakamura K, Shimai S, Kikuchi S, Tominaga K, Takahashi H, Tanaka $\mathrm{M}$, et al. Shift work and risk factors for coronary heart disease in Japanese blue-collar workers: serum lipids and anthropometric characteristics. Occup Med (Lond) 1997;47:142-6.

19. Cho YS, Kim HR, Myong JP, Kim HW. Association between work conditions and smoking in South Korea. Saf Health Work 2013;4:197-200.

20. Jung Y, Oh J, Huh S, Kawachi I. The effects of employment conditions on smoking status and smoking intensity: the analysis of Korean labor \& income panel 8(th)-10(th) wave. PLoS One 2013;8:e57109.

21. Korea Centers for Disease Control and Prevention. Korean health statistics 2012: Korea National Health and Nutrition Examination Survey (KNHANES V-3). Cheongju: Korea Centers for Disease Control and Prevention; 2013.

22. Korea Centers for Disease Control and Prevention. Korean health statistics 2011: Korea National Health and Nutrition Examination Survey 
(KNHANES V-2). Cheongju: Korea Centers for Disease Control and Prevention; 2012.

23. Korea Centers for Disease Control and Prevention. Korean health statistics 2010: Korea National Health and Nutrition Examination Survey (KNHANES V-1). Cheongju: Korea Centers for Disease Control and Prevention; 2011.

24. Bushnell PT, Colombi A, Caruso CC, Tak S. Work schedules and health behavior outcomes at a large manufacturer. Ind Health 2010;48:395405.

25. Pilcher JJ, Lambert BJ, Huffcutt AI. Differential effects of permanent and rotating shifts on self-report sleep length: a meta-analytic review. Sleep 2000;23:155-63.

26. Ministry of Gender Equality and Family. Impartiality about division of domestic duties between married couples, National Survey on Family in Korea, 2010. Seoul: Ministry of Gender Equality and Family; 2010.

27. Reid K, Dawson D. Comparing performance on a simulated 12 hour shift rotation in young and older subjects. Occup Environ Med 2001;58:58-62.

28. Torsvall L, Akerstedt T, Gillberg M. Age, sleep and irregular workhours: a field study with electroencephalographic recordings, catecholamine excretion and self-ratings. Scand J Work Environ Health 1981;7:196203.

29. Pavard B, Vladis A, Foret J, Wisner A. Age and long term shiftwork with mental load: their effects on sleep. J Hum Ergol (Tokyo) 1982;11 Suppl:303-9.

30. Gander P, Signal L. Who is too old for shift work?: developing better criteria. Chronobiol Int 2008;25:199-213. 